\title{
Influence of the Postcuring Process on Dimensional Accuracy and Seating of 3D-Printed Polymeric Fixed Prostheses
}

\author{
Jaewon Kim ${ }^{1}$ and Du-Hyeong Lee $\mathbb{D}^{2}$ \\ ${ }^{1}$ Department of Periodontics and Endodontics, School of Dental Medicine, University at Buffalo, New York, USA \\ ${ }^{2}$ Department of Prosthodontics, School of Dentistry, Institute for Translational Research in Dentistry, \\ Kyungpook National University, Daegu 41940, Republic of Korea \\ Correspondence should be addressed to Du-Hyeong Lee; deweylee@knu.ac.kr
}

Received 17 June 2020; Revised 17 September 2020; Accepted 9 November 2020; Published 16 November 2020

Academic Editor: Xiaojun Duan

Copyright (c) 2020 Jaewon Kim and Du-Hyeong Lee. This is an open access article distributed under the Creative Commons Attribution License, which permits unrestricted use, distribution, and reproduction in any medium, provided the original work is properly cited.

\begin{abstract}
The postcuring process is essential for 3-dimensional (3D) printing of photopolymer-based dental prostheses. However, the deformation of prostheses resulting from the postcuring process has not been fully investigated. The purpose of this study was to evaluate the effects of different postcuring methods on the fit and dimensional accuracy of 3D-printed full-arch polymeric fixed prostheses. A study stone model with four prosthetic implant abutments was prepared. A full-arch fixed dental prosthesis was designed, and the design was transferred to dental computer-aided manufacturing (CAM) software in which supports were designed to the surface of the prosthesis design for 3D printing. Using a biocompatible photopolymer and a stereolithography apparatus $3 \mathrm{D}$ printer, polymeric prostheses were produced $(N=21)$. In postcuring, the printed prostheses were polymerized in three different ways: the prosthesis alone, the prosthesis with supports, or the prosthesis on a stone model. Geometric accuracy of 3D-printed prostheses, marginal gap, internal gap, and intermolar distance was evaluated using microscopy and digital techniques. Kruskal-Wallis and Mann-Whitney $U$ tests with Bonferroni correction were used for the comparison of results among groups $(\alpha=0.05)$. In general, the mean marginal and internal gaps of cured prostheses were the smallest when the printed prostheses were cured with seating on the stone model $(P<0.05)$. With regard to the adaptation accuracy, the presence of supports during the postcuring process did not make a significant difference. Error in the intermolar distance was significantly smaller in the model seating condition than in the other conditions $(P<0.001)$. Seating 3D-printed prosthesis on the stone model reduces adverse deformation in the postcuring process, thereby enabling the fabrication of prostheses with favorable adaptation.
\end{abstract}

\section{Introduction}

Interim fixed dental prostheses are usually made in clinics with autopolymerizing acrylic resins $[1,2]$. This conventional manual methodology is still the mainstream approach in fabricating fixed prosthodontics but is labor-intensive and uncomfortable for patients because the direct fabrication of prostheses is performed inside the patient's mouth, and heating occurs during polymerization. Because of these drawbacks, digital scanning and computer-aided design/computer-aided manufacturing (CAD/CAM) technologies are increasingly being used to fabricate interim polymeric prostheses $[3,4]$. The oral anatomic shape is virtually registered using an optical scanner, and the scan data are imported into dedicated dental CAD software, in which the cementation space of the prosthetic crown is set and a final prosthesis is designed [5]. The design is then transferred to CAM software where the $3 \mathrm{D}$ image is divided into $2 \mathrm{D}$ cross-sectional images and processed to the polymeric prosthesis using additive manufacturing technologies [6]. The final fabrication process is the postcuring treatment of the printed prosthesis [6].

The $3 \mathrm{D}$ printing technologies have diversified treatment procedures and have become an alternative to manual and subtractive methods in medical and dental fields [7-10]. There are several different ways to print polymeric 
prostheses, such as stereolithography apparatus (SLA), digital light processing, fused deposition modeling, and polymer jetting $[11,12]$. The SLA printing method uses liquid photopolymer, and objects are built layer-by-layer using sitespecific polymerization by an ultraviolet laser [13]. For the production of interim dental prostheses via SLA printing, several photocurable resins are available and approved for long-term intraoral use $[6,14]$. Commonly used acrylic resins could be cytotoxic to the human body in the uncured state [15], but the biocompatibility significantly improves after postcuring and cleaning in the $3 \mathrm{D}$-printed objects $[6$, 16]. The quality of $3 \mathrm{D}$-printed objects significantly varies depending on operational parameters, fabrication workflow, materials, and devices [16, 17]. The contemporary 3Dprinted polymers for interim dental applications exhibit low anisotropy and appropriate properties required for the end product $[6,14]$. The mechanical and physicochemical properties of photopolymers available on the market are reported to be comparable to those of conventional autopolymerizing acrylic resins $[6,8]$.

The postcuring process increases the degree of polymerization of the printed object, which affects the final mechanical properties and the amount of the object's residual monomer $[6,14,16]$. However, postcuring can also cause dimensional deformation in the general structure and warping in any thin areas of an object because of the inherent change of the chemical bonds during polymerization [18]. Although the postcuring process is essential for the photopolymer-based 3D printing of dental prostheses, whether or not different postcuring methods affect the geometric accuracy of the prosthesis has not been fully investigated. The purpose of this study was to evaluate the effects of different postcuring conditions on the geometric accuracy of fabrication of full-arch polymeric fixed prostheses that were created using SLA 3D printing. The adaptation of prostheses on abutments and dimensional deformation were assessed by means of a marginal gap, internal gap, and intermolar distance. The null hypothesis was that the differences in the postcuring methods for printed polymeric prostheses would not affect the accuracy of their fabrication.

\section{Materials and Methods}

2.1. Fabrication of the Study Model and Full-Arch Polymeric Prostheses. The overall study procedure is described in Figure 1. The edentulous study stone model was prepared, with four prosthetic implant abutments (FreeForm ST; Osstem, Seoul, Korea) that were connected to implants (USII; Osstem) placed in the canine and second premolar areas. A virtual model was created by digitizing the surface of the stone model using a laboratory-based scanner (IDC S1; Amann Girrbach, Koblach, Austria) and was transferred to a dental design software program (R2CAD; MegaGen, Daegu, Korea), which designed a 12-unit implantsupported fixed dental prosthesis (Figure 2). The design file was transferred to a CAM software program for 3D printing (Raydent Studio; Ray, Hwaseong-si, Korea), in which supports were installed on the occlusal surface of the prosthesis design (Figure 3). Subsequently, interim acrylic prostheses

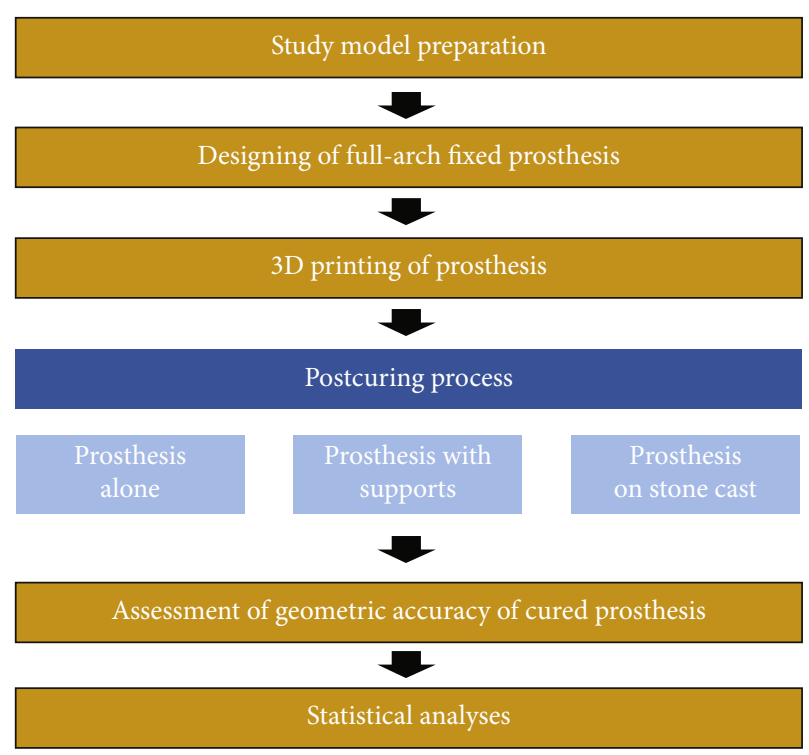

Figure 1: Workflow of this study.

were produced by printing a biocompatible photopolymer (Raydent C\&B; Ray) in a SLA 3D printer (Meg-Printer II; MegaGen) with a layering thickness of $50 \mu \mathrm{m}$ and with a wavelength of $405 \mathrm{~nm}$ (Table 1). The printed prostheses were then rinsed thoroughly under running water and spray dried at room temperature according to the manufacturer's instructions.

2.2. Postcuring Process of $3 D$-Printed Prostheses. In the postcuring procedure, the printed prostheses were polymerized in an ultraviolet curing unit of the 3D printer for 15 minutes with a wavelength of $395 \mathrm{~nm}$ and radiation power at $60 \mathrm{~mW} / \mathrm{cm}^{2}$. Three different methods were used for the postcuring procedure ( $n=7$ in each group; $N=21)$ (Figure 4 ): prosthesis alone (P group), prosthesis with supports (PS group), and prosthesis on the stone model (PM group). In the $\mathrm{P}$ group, supports were removed from the printed prosthesis using a cutter, and postcuring was performed. In the PS group, the prosthesis was cured without removing the supports. In the PM group, supports were removed, and the printed prosthesis was seated on the prosthetic abutments of a stone model, and then, the postcuring was performed. For random sampling, the printed prostheses were allocated to each group consecutively in the order of fabrication. All $3 \mathrm{D}$ printing and postcuring processes were performed by a single operator (D.H.L.).

2.3. Evaluation of Fabrication Accuracy of Prostheses. The geometric accuracy of 3D-printed prostheses was evaluated using a vertical marginal gap, internal gap, and intermolar distance. For the marginal gap assessment, the cured prosthesis was passively fitted on the stone model, and the model with prosthesis was positioned perpendicular to the table of the stereomicroscope (EGVM-452M; EG Tech, Seoul, Korea) using utility wax. The midbuccal and lingual margin areas in all abutments were then imaged three times at a magnification of $60 \mathrm{x}$ with the stereomicroscope, and each value was 


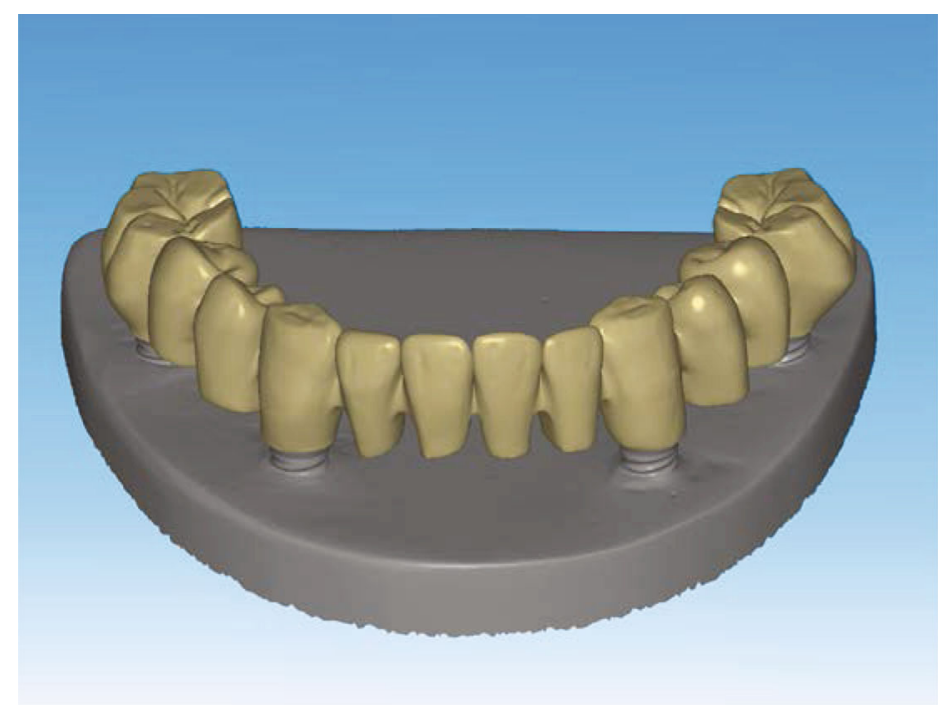

FIgURe 2: Design of implant-supported full-arch fixed dental prosthesis.

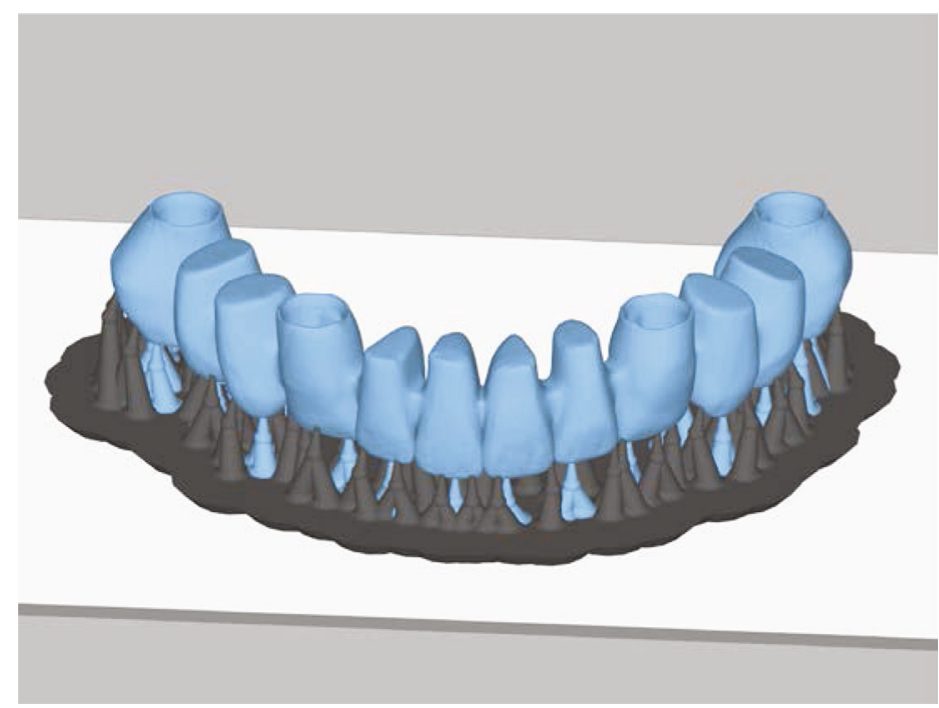

FIGURE 3: Insertion of support structures into the prosthesis design for the 3D printing procedure.

TABLE 1: Composition of photopolymer used*.

\begin{tabular}{|c|c|c|}
\hline Component & CAS No. & $\%$ \\
\hline$\alpha, \alpha^{\prime}$-[(1-Methylethylidene)di-4,1-phenylene]bis[ $\omega$-[(2-methyl-1-oxo-2-propenyl)oxy]poly(oxy-1,2-ethanediyl) & $41637-38-1$ & $20 \sim 35$ \\
\hline 7,7,9(or 7,9,9)-Trimethyl-4,13-dioxo-3,14-dioxa-5,12-diazahexadecane-1,16-diyl 2-methyl-2-propenoate & 72869-86-4 & $20 \sim 28$ \\
\hline 2-Methyl-2-propenoic acid 1,2-ethanediylbis(oxy-2,1-ethanediyl) ester & $109-16-0$ & $20 \sim 25$ \\
\hline Phenylbis(2,4,6-trimethylbenzoyl)phosphine oxide & $162881-26-7$ & $1 \sim 10$ \\
\hline Rutile $\left(\mathrm{TiO}_{2}\right)$ & 1317-80-2 & $0.1 \sim 5$ \\
\hline
\end{tabular}

${ }^{*}$ Manufacturer's information.

determined by averaging three measurements. The measurement value was defined as the vertical marginal discrepancy that was vertical marginal misfit measured parallel to the path of draw of the prosthesis [19]. For the internal gap assessment, a triple-scan technique was used, with three digital scans taken using a structured light scanner (Breuck- mann SmartScan; AICON 3D Systems GmbH, Braunschweig, Germany) [5]. The first scan was of the cured prosthesis alone, the second was of the study model, and the third was of the prosthesis on the study model. The data of the three scans were delivered to an image analysis software program (Geomagic DesignX; 3D Systems, Rock Hill, SC, 


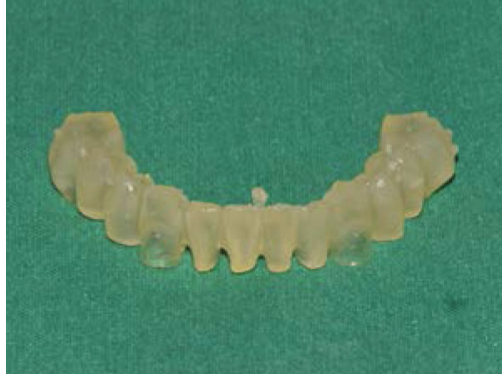

(a)

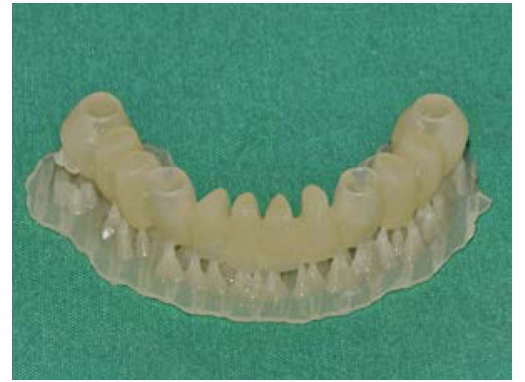

(b)

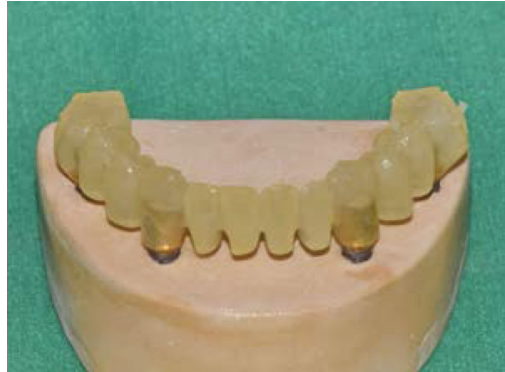

(c)

Figure 4: Postcuring methods: (a) prosthesis alone, (b) prosthesis with supports, and (c) prosthesis on the stone model.

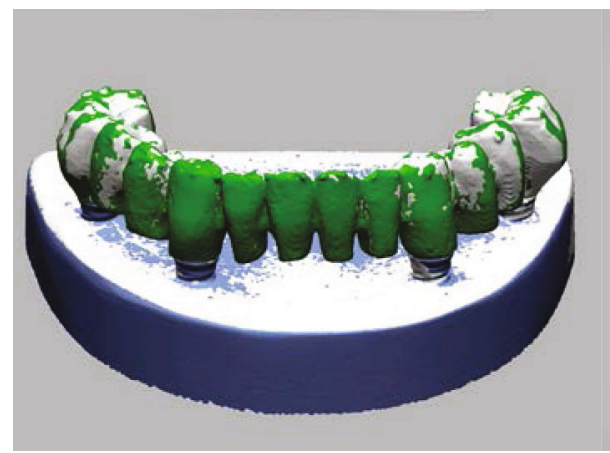

(a)

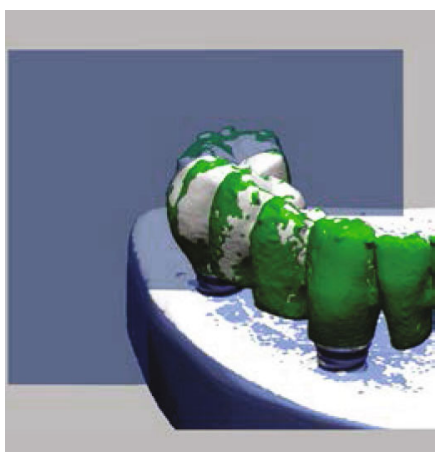

(b)

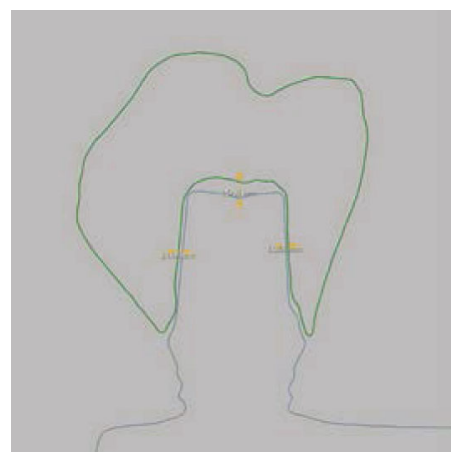

(c)

FIGURE 5: The triple scan technique for assessing the internal gap of the 3D-printed prosthesis: (a) image matching of scans, (b) measurement plane formation, and (c) cross-sectional image in the abutment area.

USA), where the three scan images were merged using an area-designated best-fit image matching (Figure 5(a)) [20]. The image alignment to the closest fit with the corresponding images was enabled using an iterative closest point (ICP) algorithm [21]. The cross-sectional line images were buccolingually obtained at the midpoint of the abutment (Figure 5(b)), and the internal gap, the perpendicular distances from the external surface of the abutment to the internal surface of the prosthesis, was measured at the center points of the buccal, lingual, and occlusal aspects (Figure 5(c)). For the intermolar distance assessment, a virtual cross-sectional plane passing through two central fossae of the first molars on both sides was created, and the distance between the most external points of the buccal surfaces of the left and right first molars was measured and compared with that of the prosthesis design image using the image analysis software program. All measurements for evaluating the accuracy of 3D-printed prostheses were carried out by a single examiner blinded to the research objective.

2.4. Statistical Analysis. All outcome variable data were reported as mean \pm standard deviation. The Kruskal-Wallis test was used for the comparison of the results among groups that used different postcuring methods using the IBM Statistical Package for the Social Sciences (SPSS) v25.0 for Windows (IBM Corp., Chicago, IL, USA). The statistical significance level was set at 0.05 . The Mann-Whitney $U$ test with Bonferroni correction was used for post hoc analyses $(\alpha=0.017)$.

\section{Results}

Table 2 presents the results of fit and dimensional discrepancy of the 3D-printed acrylic prostheses at each measurement point. In general, the PM group showed the lowest mean discrepancy, followed by the PS and P groups. The PS and P groups showed no significant difference in any measurement outcome. The highest discrepancy was found in the measurements of the occlusal area, especially in the P group. Figure 6 shows images of the marginal gap in the different postcuring groups. The PM group exhibited significantly smaller marginal gaps than the other groups. Figure 7 shows the outlines of the molars of 3D-printed prostheses and the design image in the cross-sectional view. Again, the discrepancy of intermolar distance was the smallest in the PM group.

\section{Discussion}

This study was designed to find a postcuring method that minimizes the adverse dimensional change for 3D-printed polymeric prostheses. The results showed that the adaptation and dimension of cured prostheses were most accurate when the printed prostheses were cured with seating on the stone model. Thus, the null hypothesis that the differences in the postcuring methods for printed polymeric prostheses would 
TABLE 2: Discrepancy values (mean \pm standard deviation; $\mu \mathrm{m}$ ) of 3D-printed polymeric fixed prostheses fabricated by different postcuring methods.

\begin{tabular}{|c|c|c|c|c|}
\hline Area & Prosthesis alone & $\begin{array}{l}\text { Postcuring meth } \\
\text { Prosthesis with supports }\end{array}$ & Prosthesis on the stone model & $P$ \\
\hline Margin, buccal & $274.4 \pm 64.4^{\mathrm{a}}$ & $233.0 \pm 40.3^{\mathrm{a}}$ & $91.8 \pm 27.4^{\mathrm{b}}$ & 0.008 \\
\hline Axial, buccal & $122.1 \pm 51.6$ & $125.4 \pm 47.8$ & $94.7 \pm 64.5$ & 0.468 \\
\hline Occlusal & $332.8 \pm 70.7^{\mathrm{a}}$ & $311.7 \pm 58.2^{\mathrm{a}}$ & $126.3 \pm 27.3^{\mathrm{b}}$ & 0.009 \\
\hline Axial, lingual & $138.6 \pm 46.7^{\mathrm{a}}$ & $134.3 \pm 28.4^{\mathrm{a}}$ & $64.4 \pm 22.2^{\mathrm{b}}$ & 0.019 \\
\hline Margin, lingual & $256.4 \pm 46.2^{\mathrm{a}}$ & $196.8 \pm 38.7^{\mathrm{a}}$ & $89.0 \pm 26.7^{b}$ & 0.004 \\
\hline Intermolar & $115.4 \pm 25.3^{\mathrm{a}}$ & $105.9 \pm 12.9^{\mathrm{a}}$ & $39.2 \pm 17.7^{\mathrm{b}}$ & 0.008 \\
\hline
\end{tabular}

Different superscript lowercase letters indicate significant differences within a row $(\alpha=0.05)$.

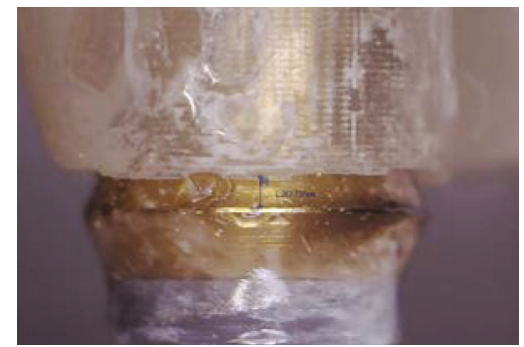

(a)

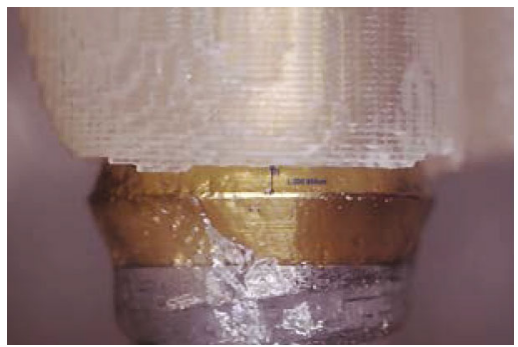

(b)

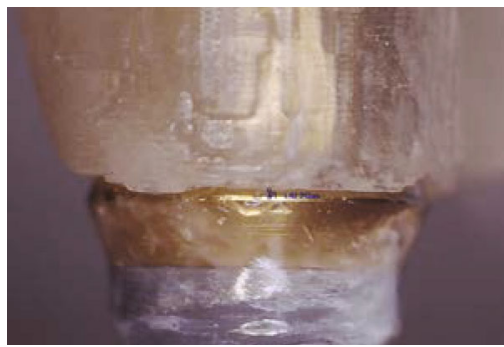

(c)

FIgURE 6: Microscopic image for marginal gap measurement: (a) prosthesis alone, (b) prosthesis with supports, and (c) prosthesis on the stone model.

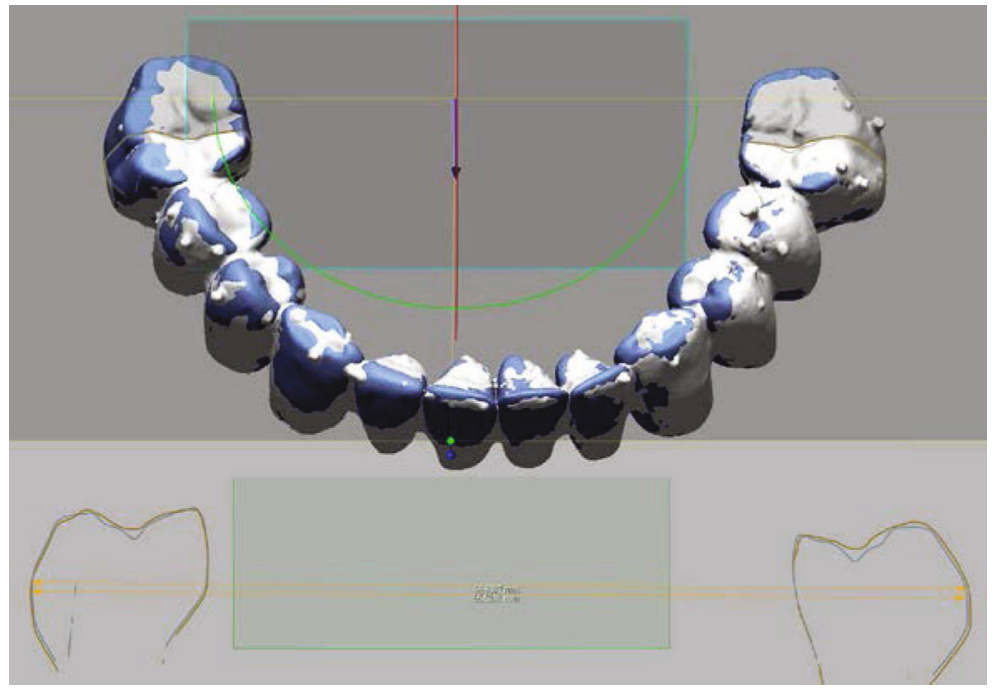

FIgURE 7: Evaluation of discrepancy of the intermolar distance between 3D-printed and design image of prosthesis.

not affect the accuracy of their fabrication was rejected. The mean marginal gap of prostheses in the PM group was lower than $120 \mu \mathrm{m}$, which was in a clinically acceptable range. The error in the intermolar distance was significantly smaller in the PM group than the other groups of postcuring without the model. Leaving the supports did not significantly decrease the dimensional error that happened in the postcuring process.

During the printing process, each layer is briefly exposed to curing light and partially solidified. This incomplete poly- merization is needed to allow fusion between layers [22]. After the printing process, postcuring is performed to achieve the maximum strength and full density of the material [23]. Chemically, the polymerization of resin material is the increase of the conversion degree, which is a chemical structure change from carbon double bonds $(\mathrm{C}=\mathrm{C})$ to carbon single (C-C) bonds [24]. The change in chemical structure inevitably involves a dimensional change of the object $[23,25]$. Accordingly, the amount of deformation in postcuring could be affected by the change in the conversion 
degree. The findings of the present study showed that deformation during postcuring could be minimized when the $3 \mathrm{D}$-printed prosthesis was seated on the abutments of the stone model, leading to markedly low misfit and dimensional error. This might happen because the underlying abutments played the role of mechanical guides that blocked unwanted deformation. Therefore, it is recommended that 3D-printed prostheses are placed on the abutments of the model during the postcuring process. This method may help fabricate prostheses that are closely fitted to abutments in the mouth, in addition to less cement leakage, less need for occlusal adjustment, and better performance of the prosthesis in the long term [26].

The novelty of this study is that it is the first to investigate the impact of postcuring methods on dimensional accuracy in the 3D printing of full-arch polymeric fixed prostheses. Although the design of this study was controlled, there are several limitations derived from its in vitro nature. Comprehensive clinical studies including tooth-supported and implant-supported conditions are necessary, and the clinical marginal and internal fit of prostheses needs to be assessed to confirm the findings of the present study. In addition, the deformation phenomenon that occurs during the postcuring process should be evaluated using different $3 \mathrm{D}$ printing methods, such as digital light processing, fused deposition modeling, and polymer jetting. Photopolymers are composed of oligomers, monomers, and photoinitiators, and the curing of photopolymers is affected by wavelength, power of light, and radiation time. Thus, further studies on materials and curing setting are needed to optimize the postcuring process.

\section{Conclusions}

Within the limitations of this study, the postcuring process affects the fit and dimensional accuracy of 3D-printed polymeric prostheses. Seating of the prosthesis on the stone model is recommended to minimize the deformity of the prosthesis during the postcuring process.

\section{Data Availability}

The data used to support the findings of this study are included within the article.

\section{Conflicts of Interest}

The authors declare that there is no conflict of interest regarding the publication of this paper.

\section{Authors' Contributions}

J Kim contributed to the conceptualization of this study, data curation, formal analysis, investigation, and original drafting. DH Lee was involved in the methodology, formal analysis, investigation, review, editing, and supervision of this study.

\section{Acknowledgments}

The authors thank Yong-Do Choi for help with the laboratory work.

\section{References}

[1] D. R. Burns, D. A. Beck, and S. K. Nelson, “A review of selected dental literature on contemporary provisional fixed prosthodontic treatment: report of the Committee on Research in Fixed Prosthodontics of the Academy of Fixed Prosthodontics," Journal of Prosthetic Dentistry, vol. 90, no. 5, pp. 474497, 2003.

[2] D. G. Gratton and S. A. Aquilino, "Interim restorations," Dental Clinics of North America, vol. 48, no. 2, pp. 487-497, 2004.

[3] F. Mangano and G. Veronesi, "Digital versus analog procedures for the prosthetic restoration of single implants: a randomized controlled trial with 1 year of follow-up," BioMed Research International, vol. 2018, Article ID 5325032, 20 pages, 2018.

[4] N. Alharbi, S. Alharbi, V. Cuijpers, R. B. Osman, and D. Wismeijer, "Three-dimensional evaluation of marginal and internal fit of 3D-printed interim restorations fabricated on different finish line designs," Journal of Prosthodontic Research, vol. 62, no. 2, pp. 218-226, 2018.

[5] M. Kim, J. Kim, H. N. Mai et al., "Comparative clinical study of the marginal discrepancy of fixed dental prosthesis fabricated by the milling-sintering method using a presintered alloy," The Journal of Advanced of Prosthodontics, vol. 11, no. 5, pp. 280-285, 2019.

[6] M. Revilla-León, M. J. Meyers, A. Zandinejad, and M. Özcan, "A review on chemical composition, mechanical properties, and manufacturing work flow of additively manufactured current polymers for interim dental restorations," Journal of Esthetic and Restorative Dentistry, vol. 31, no. 1, pp. 51-57, 2019.

[7] X. J. Duan, H. Q. Fan, F. Y. Wang, P. He, and L. Yang, "Application of 3D-printed customized guides in subtalar joint arthrodesis," Orthopaedic Surgery, vol. 11, no. 3, pp. 405413, 2019.

[8] A. Tahayeri, M. Morgan, A. P. Fugolin et al., "3D printed versus conventionally cured provisional crown and bridge dental materials," Dental Materials, vol. 34, no. 2, pp. 192-200, 2018.

[9] H. N. Mai, K. B. Lee, and D. H. Lee, "Fit of interim crowns fabricated using photopolymer-jetting 3D printing," Journal of Prosthetic Dentistry, vol. 118, no. 2, pp. 208-215, 2017.

[10] C. Polzin, S. Spath, and H. Seitz, "Characterization and evaluation of a PMMA-based 3D printing process," Rapid Prototyping Journal, vol. 19, no. 1, pp. 37-43, 2013.

[11] M. Revilla-León, M. J. Meyer, A. Zandinejad, and M. Özcan, "Additive manufacturing technologies for processing zirconia in dental applications," International journal of computerized dentistry, vol. 23, no. 1, pp. 27-37, 2020.

[12] R. van Noort, "The future of dental devices is digital," Dental Materials, vol. 28, no. 1, pp. 3-12, 2012.

[13] J. W. Stansbury and M. J. Idacavage, "3D printing with polymers: challenges among expanding options and opportunities," Dental Materials, vol. 32, no. 1, pp. 54-64, 2016.

[14] J. Jockusch and M. Özcan, "Additive manufacturing of dental polymers: an overview on processes, materials and applications," Dental Materials Journal, vol. 39, no. 3, pp. 345-354, 2020.

[15] A. Bagheri and J. Jin, "Photopolymerization in 3D printing," ACS Applied Polymer Materials, vol. 1, no. 4, pp. 593-611, 2019.

[16] F. Alifui-Segbaya, "Biomedical photopolymers in 3D printing," Rapid Prototyping Journal, vol. 26, no. 2, pp. 437-444, 2019. 
[17] N. Alharbi, R. Osman, and D. Wismeijer, "Effects of build direction on the mechanical properties of 3D-printed complete coverage interim dental restorations," Journal of Prosthetic Dentistry, vol. 115, no. 6, pp. 760-767, 2016.

[18] J. R. Dizon, A. Espera, Q. Chen, and R. Advincula, "Mechanical characterization of 3D-printed polymers," Additive Manufacturing, vol. 20, pp. 44-67, 2017.

[19] J. R. Holmes, S. C. Bayne, G. A. Holland, and W. D. Sulik, "Considerations in measurement of marginal fit," Journal of Prosthetic Dentistry, vol. 62, no. 4, pp. 405-408, 1989.

[20] S. Holst, M. Karl, M. Wichmann, and R. E. Matta, "A new triple-scan protocol for 3D fit assessment of dental restorations," Quintessence International, vol. 42, no. 8, pp. 651$657,2011$.

[21] A. Almukhtar, X. Ju, B. Khambay, J. McDonald, and A. Ayoub, "Comparison of the accuracy of voxel based registration and surface based registration for 3D assessment of surgical change following orthognathic surgery," PLoS One, vol. 9, no. 4, article e93402, 2014.

[22] J. Crivello and E. Reichmanis, "Photopolymer materials and processes for advanced technologies," Chemistry of Materials, vol. 26, pp. 533-548, 2013.

[23] K. Yoshida and E. H. Greener, "Effect of photoinitiator on degree of conversion of unfilled light-cured resin," Journal of Dentistry, vol. 22, no. 5, pp. 296-299, 1994.

[24] D. H. Lee, H. N. Mai, J. C. Yang, and T. Y. Kwon, "The effect of 4,4'-bis(N,N-diethylamino) benzophenone on the degree of conversion in liquid photopolymer for dental 3D printing," The Journal of Advanced of Prosthodontics, vol. 7, no. 5, pp. 386-391, 2015.

[25] M. Balkenhol, M. Knapp, P. Ferger, U. Heun, and B. Wöstmann, "Correlation between polymerization shrinkage and marginal fit of temporary crowns," Dental Materials, vol. 24, no. 11, pp. 1575-1584, 2008.

[26] D. Ehrenberg, G. I. Weiner, and S. Weiner, "Long-term effects of storage and thermal cycling on the marginal adaptation of provisional resin crowns: a pilot study," Journal of Prosthetic Dentistry, vol. 95, no. 3, pp. 230-236, 2006. 